\title{
Disappearance of Vortex Lift in Low-Aspect-Ratio Wings at Very-low Reynolds Numbers*
}

\author{
Masato OKAмото, ${ }^{\dagger}$ Daisuke SASAKI, Masaya KAMIKUBO, and Ryota FuJII \\ Department of Aeronautics, Kanazawa Institute of Technology, Hakusan, Ishikawa 924-0838, Japan
}

\begin{abstract}
The objective of this study is to ascertain the aerodynamic characteristics of low-aspect-ratio wings at Reynolds numbers ranging between $1 \times 10^{3}$ and $1 \times 10^{4}$ which correspond to insect wings. The wings tested in this study are rectangular, thin, flat plates with aspect ratios varying from 0.5 to 2 . The very small forces and moment acting on the wings were measured using a low-pressure wind tunnel. Although a large maximum lift coefficient was obtained for the wing with an aspect ratio of 1 at a Reynolds number of $1 \times 10^{4}$, it decreased as the Reynolds number decreased due to the disappearance of vortex lift. That is, the Reynolds number effects of low-aspect-ratio wings were found at the current Reynolds number. However, as the aspect ratio of the wing changed, the rate at which the maximum lift coefficient decreased was reduced. The main cause of the phenomenon was the behavior of the wing-tip vortices. These aerodynamic characteristics of wings will be important for developing insect-sized aircraft.
\end{abstract}

Key Words: Aerodynamic Characteristics, Wing, Low Reynolds Number, Wind Tunnel Test

\section{Introduction}

Recently, the study of low Reynolds number aircraft such as micro-aerial vehicles (MAVs) has increased. Further, smaller aircrafts performing at low Reynolds numbers corresponding to insect wings have been proposed. Pines and Bohorquez $^{1)}$ and Wood et al. ${ }^{2)}$ mentioned the importance of investigating the aerodynamics and morphological characteristics of flying creatures with Reynolds numbers $(R e)$ of $R e<4 \times 10^{4}$. Furthermore, research institutions such as JAXA and others ${ }^{3)}$ are planning to launch Mars exploratory unmanned aircraft that performs at low Reynolds number because the air density of Mars is 1/100 that of Earth.

Several groups, such as Okamoto et al., ${ }^{4)}$ Azuma et al., ${ }^{5)}$ Kesel, ${ }^{6)}$ Sunada et al., ${ }^{7)}$ Obata and Shinohara, ${ }^{8)}$ Shi et al. ${ }^{9}$ and Okamoto and Ebina, ${ }^{10}$ have investigated the twodimensional characteristics of steady wings experimentally (at $R e \leq 1 \times 10^{4}$ ) using both wind tunnel and water tank tests. These studies arrived at the following conclusions: i) Effective airfoils designed for low Reynolds numbers are thin, have a sharp leading-edge, and a corrugated profile, similar to the dragonfly wing. On the other hand, streamlined airfoils having large thickness and a rounded leading-edge, which are used in large aircraft, exhibit poor performance due to laminar separation in low Reynolds number flows. ii) The lift curves of low Reynolds number airfoils show nonlinearity due to a separation bubble. ${ }^{11)}$ Therefore, the aerodynamic characteristics of two-dimensional wings strongly depend on the Reynolds number.

On the other hand, the discussion of aerodynamic characteristics of three-dimensional wings is also important to dis-

(C) 2019 The Japan Society for Aeronautical and Space Sciences

*Received 4 February 2019; final revision received 20 April 2019; accepted for publication 18 July 2019.

†Corresponding author, okmt@neptune.kanazawa-it.ac.jp tinguish between two-dimensional and three-dimensional wings. This is because the aerodynamic characteristics of large-aspect-ratio wings are strongly affected by the wing section profile, whereas those of low-aspect-ratio wings are affected by the wing planforms.

Low-aspect-ratio wings of nearly 1 are strongly affected by the wing planform. Torres and Mueller ${ }^{12)}$ studied elliptical and rectangular wing planforms with aspect ratios of $A R=0.5-2$ by conducting wind tunnel testing at $R e=7 \times 10^{4}-10 \times 10^{4}$ to develop MAVs. Okamoto and Azuma ${ }^{13)}$ investigated the aerodynamic characteristics of flat plates having a wide range of aspect ratios $(0.5 \leq A R \leq 8)$ at $R e=1 \times 10^{4}-2 \times 10^{4}$. Mizoguchi and Itoh ${ }^{14)}$ investigated a low-aspect-ratio $(A R=1)$ flat plate at $R e=$ $5.2 \times 10^{4}-7.6 \times 10^{4}$ in detail. In both experimental studies, the results were compared with the theoretical lift slope of low-aspect-ratio wings based on the lifting surface theory, such as the vortex lattice method (VLM), by applying Polhamus' suction analogy ${ }^{15,16)}$ to simulate the vortex lift by the tip vortex. The Reynolds number effects of low-aspect-ratio wings seem to be comparatively small based on the results of these studies.

However, preceding the present study, we conducted lowpressure wind tunnel tests ${ }^{17)}$ and found that the vortex lift of low-aspect-ratio wings disappeared in the lift slope of a rectangular flat plate with $A R=1$ when the aerodynamic characteristics of the wing were examined at $R e \leq 1.5 \times 10^{4}$. This result was further verified by computational fluid dynamics (CFD). ${ }^{18)}$ The Reynolds number effects of the aerodynamic characteristics of low-aspect-ratio wings existed at a very-low Reynolds number corresponding to an insectsized wing. Furthermore, this phenomenon seems to hold in wings having other planforms. ${ }^{19)}$ The present study contains detailed experimental results from rectangular wings. The aerodynamic characteristics of rectangular wings with 
several aspect ratios $(0.5 \leq A R \leq 2)$ at $1 \times 10^{3} \leq R e \leq$ $1 \times 10^{4}$ are discussed in this paper. These aerodynamic characteristics of wings will be important for developing insectsized aircraft.

\section{Materials and Methods}

A low-pressure wind tunnel, ${ }^{17)}$ which is a very small pusher-type wind tunnel installed in a low-pressure chamber made of acrylic plate, was used to measure the very small forces and moment acting on a small wing in very-low Reynolds number flows. The size of the rectangular test section in the wind tunnel was $100 \mathrm{~mm}(\mathrm{H}) \times 160 \mathrm{~mm}(\mathrm{~W}) \times$ $180 \mathrm{~mm}(\mathrm{~L})$. The wind velocity $U$ at the test section could be regulated from 2 to $10 \mathrm{~m} / \mathrm{s}$ in each low-pressure environment between 0.1 and $1 \mathrm{~atm}$. The turbulence intensity along the wind velocity was less than $0.3 \%(\sqrt{\bar{u}} / U<0.3 \%)$ at $U \geq 3 \mathrm{~m} / \mathrm{s}$ under atmospheric pressure, and it increased as the wind velocity decreased. Although the turbulence intensity under a low-pressure environment was unknown, the wind velocity was made as large as possible by setting the low-pressure environment as the Reynolds number decreased. The present study was conducted under atmospheric pressure for the measurement at $R e=1 \times 10^{4}$, and at 0.1 atm for $R e=1 \times 10^{3}$. Regarding the setup of this wind tunnel, please refer to Ref. 17) for more details.

The lift $L$, drag $D$, and pitching moment about $25 \%$ chord $M, 0.25$ c were measured using the original load measuring device. The angle of attack $\alpha$ of the wing model was varied from $-15 \mathrm{deg}$ to $+60 \mathrm{deg}$ by the driving mechanism of a stepping motor controlled by a microcomputer. The output signals of the measuring device were analyzed using a personal computer through a bridge circuit, a strain amplifier, and a 16-bit analog-to-digital converter. The resolutions of balance outputs for the lift, drag, and pitching moment were $3.2 \times 10^{-2} \mathrm{mN}, 2.6 \times 10^{-2} \mathrm{mN}$, and $5.3 \times 10^{-4} \mathrm{mNm}$, respectively. The maximum bias errors in the outputs were less than $0.1 \%$ and $0.5 \%$, respectively, for the forces and moment over the full load range $\left(0.2 \mathrm{~N}\right.$ and $\left.3 \times 10^{-3} \mathrm{Nm}\right)$. The aerodynamic data were obtained by averaging 300 datasets measured at a sampling frequency of $100 \mathrm{~Hz}$. The output data obtained in an averaging time of $3 \mathrm{~s}$ were confirmed to have sufficient repeatability.

Figure 1 shows a test section of the wind tunnel. The three-dimensional wing was supported by the strut. The aerodynamic forces and moment acting on the strut were measured previously using a dummy wing model supported at other point of the test section in front of the strut. The aerodynamic forces and moment acting on the wing model were determined by subtracting those acting on the strut (subscript st) from the data measured (subscript m) as follows:

$$
L=L_{\mathrm{m}}-L_{\mathrm{st}}, \quad D=D_{\mathrm{m}}-D_{\mathrm{st}}, \quad M=M_{\mathrm{m}}-M_{\mathrm{st}} .
$$

The non-dimensional aerodynamic coefficients of the wing (i.e., lift coefficient $C_{L}$, drag coefficient $C_{D}$, and pitching moment coefficient $C_{M, 0.25 c}$ ) were obtained by normalizing with the dynamic pressure $q=\rho U^{2} / 2$ ( $\rho$ : air density),

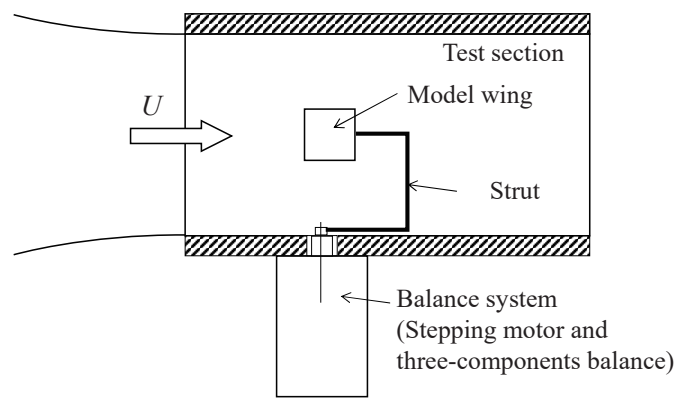

Fig. 1. Model wing arrangement in the test section of wind tunnel.

and the model wing area $S$ for forces and $S c$ for moment as follows:

$$
C_{L}=L / q S, \quad C_{D}=D / q S, \quad C_{M, 0.25 c}=M_{, 0.25 c} / q S c .
$$

Here, a positive $C_{M, 0.25 c}$ represents the nose-up moment.

The variations in the coefficients measured were small because the standard deviation was less than 0.005 for each coefficient; each standard deviation being obtained from 40 datasets measured at a large angle of attack. The uncertainties analyzed from these datasets were $0.1 \%$ of the mean value for $C_{L}$ and $C_{D}$, and $1 \%$ for $C_{M, 0.25 c}$. However, the factors of experimental conditions in a low-pressure environment were not included in these uncertainties. Accordingly, the consistency of the data obtained in different pressure environments was confirmed.

The aerodynamic coefficients measured may be affected by the blockage caused by the walls of the test section. Therefore, a correction method ${ }^{20)}$ that is useful for flow separation was used for this study. This correction method was previously verified by examining a larger test section of the wind tunnel, and was used in a previous study. ${ }^{13)}$ The dynamic pressure was corrected by introducing a blockage correction factor $\varepsilon$, expressed by the following equation:

$$
\varepsilon=\frac{\Delta u}{U}=\frac{S}{4 S_{t s}} C_{D 0}+\frac{K_{W} S}{4 S_{t s}}\left(C_{D}-C_{D 0}-C_{D i}\right) .
$$

Here, $S / S_{t s}$ is the ratio of the wing area $S$ to the test section area $S_{t s} . K_{W}(=5)$ is a constant in the present wing model that depends on the wing aspect ratio. $C_{D 0}$ is the minimum drag coefficient and $C_{D i}$ is the drag coefficient induced given by $C_{L}^{2} / \pi A R$, and $C_{D}-C_{D 0}-C_{D i}$, which is the second term in Eq. (3), is the drag coefficient due to the flow separation. Therefore, a larger $\varepsilon$ will be obtained for a larger $C_{D}$ at a higher angle of attack. The corrected dynamic pressure $q_{c}$ is given by:

$$
q_{c}=q(1+\varepsilon)^{2} \text {. }
$$

Because $S / S_{t s}$ was up to $5 \%$ in the wing models used in this study, the resulting $C_{L}$ had a maximum difference of $10 \%$ at $\alpha=60 \mathrm{deg}$.

Flow visualization techniques, namely the smoke-wire method and particle image velocimetry (PIV) method, were applied under atmospheric pressure to the same small wind tunnel as that used in the low-pressure experiment. Figures 2(a) and (b) show the flow visualization methods 


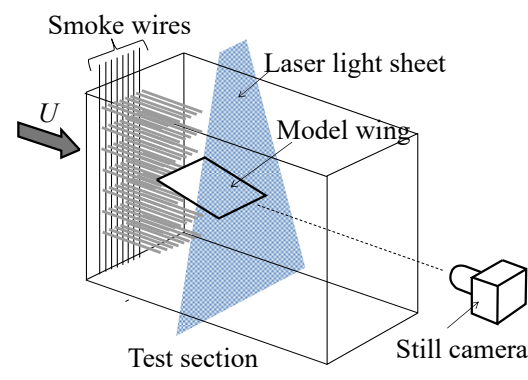

(a) Smoke-wire method

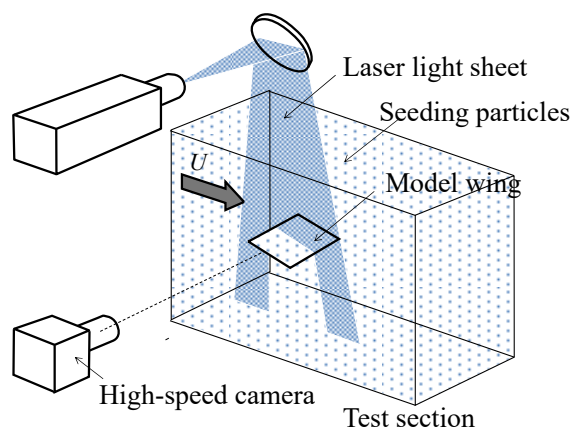

(b) PIV method

Fig. 2. Flow visualization method.

in the present study. For visualizing the tip vortex, eight smoke wires each having a $0.2-\mathrm{mm}$ diameter were stretched in front of the test section of the wind tunnel. The crosssection of the model wing tip vortex, irradiated by a laser light sheet, was taken using a still camera at a shutter speed of $1 / 250 \mathrm{~s}$. In addition, the PIV method was carried out for visualizing the chord-wise directional flow at the vertical plane of the wing. For conducting the PIV method, tracer particles of olive oil were irradiated by the laser light sheet and recorded using a high-speed camera (HLS-L2, DITECT Co., Ltd.). These video images were processed using PIV software (Flownizer2D, DITECT Co., Ltd.).

Figure 3 shows the model wings examined in the present study. The planforms of the wings were rectangular flat plates with aspect ratios varying from 0.5 to 2 . The model wings were made of thin aluminum plates having a thickness of $t=0.3 \mathrm{~mm}$ and the rectangular wing sections were cut vertically to avoid having sharp leading-edges. Table 1 shows the specifications of the model wings. The thickness ratios of the model wings changed slightly, from $1 \%$ to $1.5 \%$, according to the aspect ratios and chord length, because the wing-span was limited by the size of the test section.

\section{Results and Discussion}

\subsection{Aerodynamic characteristics of the $A R=1$ wing}

Our previous study ${ }^{17)}$ showed the aerodynamic characteristics for an $A R=1$ wing. In the present study, the wind tunnel tests of the $A R=1$ wing were repeated for comparison with wings having other aspect ratios. The results corre-

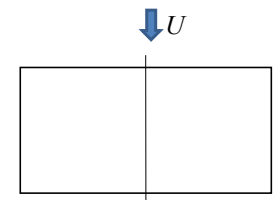

$A R=2$ wing

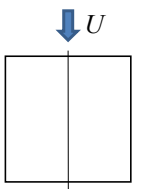

$A R=1$ wing

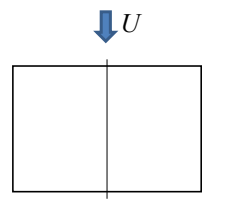

$A R=1.5$ wing

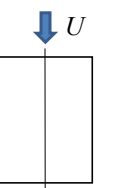

$A R=0.75$ wing

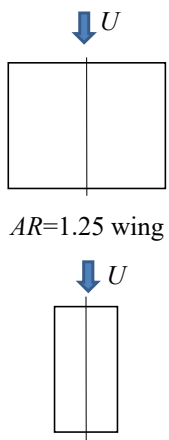

$A R=0.5$ wing
Fig. 3. Model wings tested in this study.

Table 1. Specifications of model wings.

\begin{tabular}{lccc}
\hline \multicolumn{1}{c}{ Wing model } & Chord $c(\mathrm{~mm})$ & Span $b(\mathrm{~mm})$ & Thickness $t / c(\%)$ \\
\hline$A R=2$ wing & 20 & 40 & 1.5 \\
$A R=1.5$ wing & 20 & 30 & 1.5 \\
$A R=1.25$ wing & 25 & 31.25 & 1.2 \\
$A R=1$ wing & 25 & 25 & 1.2 \\
$A R=0.75$ wing & 25 & 18.75 & 1.2 \\
$A R=0.5$ wing & 30 & 15 & 1 \\
\hline
\end{tabular}

sponded well with the previous study. Those experimental results are shown again, and an overview of the aerodynamic characteristics is described. Here, $C_{M, 0.25 c}$ of $R e=1 \times 10^{3}$ was not shown in the figures because it was too small to measure.

Figure 4 shows the aerodynamic characteristics of an $A R=1$ wing obtained by varying the Reynolds number between $1 \times 10^{3}$ and $1 \times 10^{4}$. Distinctive lift curve is seen for a low-aspect-ratio wing at $R e=1 \times 10^{4}$. Here, $C_{L}$ increases with the angle of attack $\alpha$ and decreases rapidly at $\alpha=35 \mathrm{deg}$. The continuous increase of the lift slope seen in the lift curve of the low-aspect-ratio wing is due to the additional vortex lift of the large tip vortex.

Here, the vortex lift is considered as follows: The tip vortex of the wing is the spiral vortex rolled up after leaving the sharp leading-edge of the wing. Because the flow over the tip vortex reattaches on the wing surface, the upper-surface flow of the wing flown beyond the vortex sheet is accelerated and the lift acting on the wing is further increased as the vortex lift. However, the lift curve of the smaller Reynolds number shows that the angle of attack, at which $C_{L}$ decreases rapidly, decreases as the Reynolds number decreases. Moreover, the continuous increase in $C_{L}$ is hardly noticeable at $R e=$ $1 \times 10^{3}$. This is the phenomenon disappearing vortex lift reported in this paper.

The nature of $C_{D}$ is similar to the non-linear lift curve, as it first increases with the angle of attack and then rapidly decreases. The $C_{D}$ of the flat plate changes according to Eq. (6), described later. The maximum lift-to-drag ratio $(L / D)_{\max }$ at $R e \geq 7 \times 10^{3}$ is obtained at $\alpha=7.5 \mathrm{deg}$, and the angle of attack of $(L / D)_{\max }$ increases as the Reynolds number decreases. 


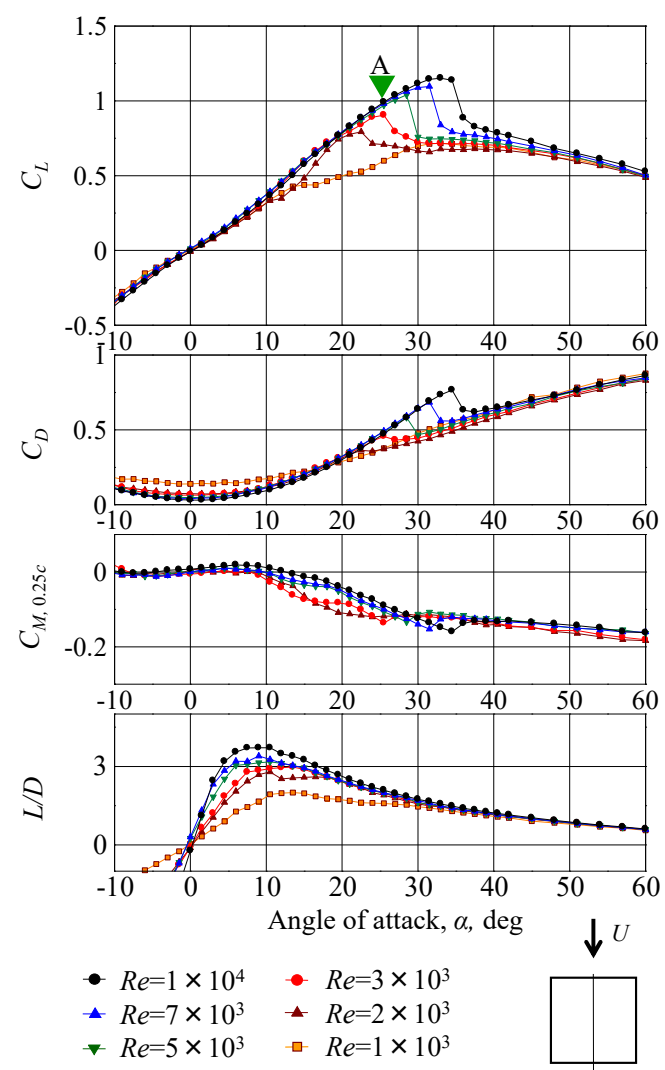

Fig. 4. Aerodynamic characteristics of $A R=1$ wing.

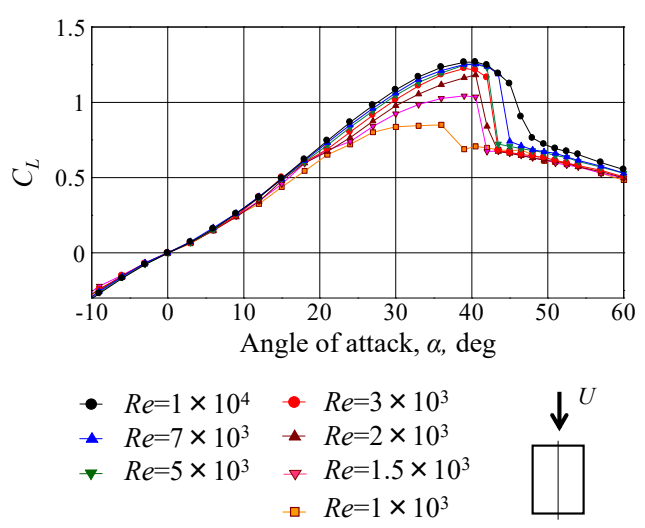

Fig. 5. Lift curves of $A R=0.75$ wing.

The slope of the $C_{M, 0.25 c}$ curve is slightly positive at $\alpha=0 \mathrm{deg}$, and becomes negative at $\alpha>8 \mathrm{deg}$. The negative slope of $C_{M, 0.25 c}$ increases as the Reynolds number decreases. As the rate of $C_{L}$ increasing shows down at a large angle of attack, the negative slope decreases again.

\subsection{Aerodynamic characteristics of the wing with} $A R<1$

Figures 5 and 6 show the aerodynamic characteristics of the wings with $A R=0.75$ and $A R=0.5$, respectively. Only the lift curves showing the most characterized coefficients are shown in Fig. 5. Similar to $A R=1$, here, the $C_{L}$ of $R e=1 \times 10^{4}$ increases as the angle of attack increases. However, $C_{L \max }(=1.27)$ is slightly larger than that of $A R=1$. As the Reynolds number decreases, the lift slope,

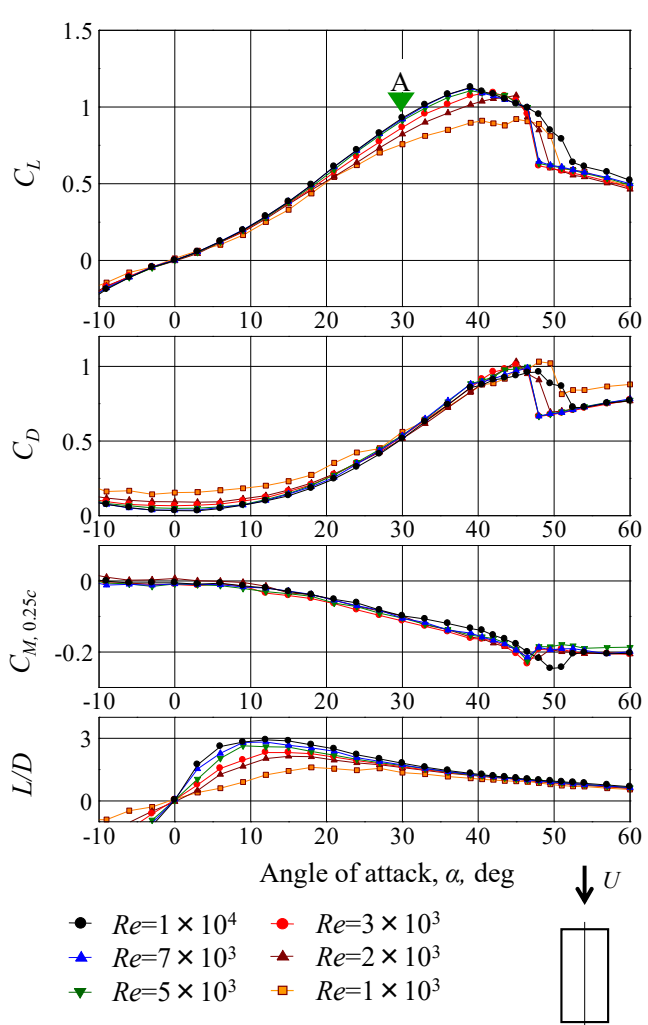

Fig. 6. Aerodynamic characteristics of $A R=0.5$ wing.

$C_{L \max }$, and the angle of attack causing a rapid decrease in $C_{L}$ gradually drops. The lift slope and $C_{L \max }$ decrease significantly at $R e<2 \times 10^{3}$.

As shown in the lift curves of the $A R=0.5$ wing in Fig. 6, $C_{L}$ increases with the smaller lift slope according to the increase in angle of attack. Although the lift slope and $C_{L \max }$ gradually decrease as the Reynolds number decreases, $C_{L \max }$ of $A R=0.5$ is larger than that of $A R=0.75$ at $R e=1 \times 10^{3}$. That is, the phenomenon of $C_{L}$ decreasing at a low Reynolds number (i.e., the $A R=1$ wing) is no longer seen in the $A R=0.5$ wing.

$(L / D)_{\max }$ decreases as the aspect ratio increases due to the increase in induced drag. Furthermore, $C_{M, 0.25 c}$ decreases as the angle of attack increases. The slight positive slope seen with the $A R=1$ wing near $\alpha=0 \mathrm{deg}$ is hardly noticeable in the $A R=0.5$ wing.

3.3. Aerodynamic characteristics of the wing with $A R>1$

Figures 7, 8, and 9 show the aerodynamic characteristics of the wings with $A R=1.25, A R=1.5$, and $A R=2$, respectively. Here, for the $A R=1.25$ and $A R=1.5$ wings, only the lift curves showing the most characterized coefficients are shown in Figs. 7 and 8. $C_{L}$ increases as the angle of attack increases until $\alpha=20 \mathrm{deg}$ at $R e \geq 7 \times 10^{3}$, and the non-linear lift slope is remarkable in comparison with that of the $A R=1$ wing. The maximum lift coefficient $C_{L \max }$ is smaller than that of the $A R=1$ wing. As the angle of attack increases beyond that of $C_{L \max }$, instead of rapidly decreasing as seen with the $A R=1$ wing, the $C_{L}$ of wings with higher aspect ratios decreases gradually. As the Reynolds number 


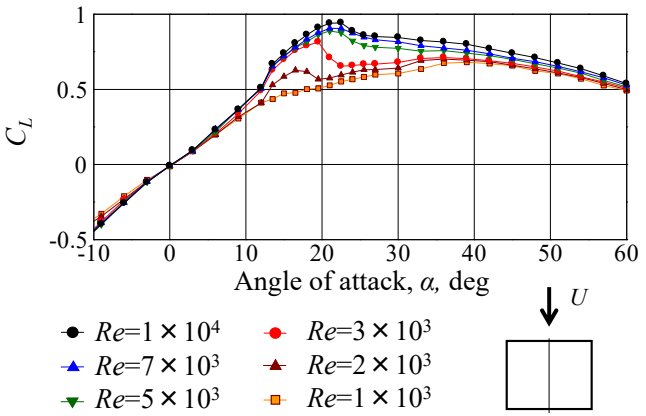

Fig. 7. Lift curves of $A R=1.25$ wing.

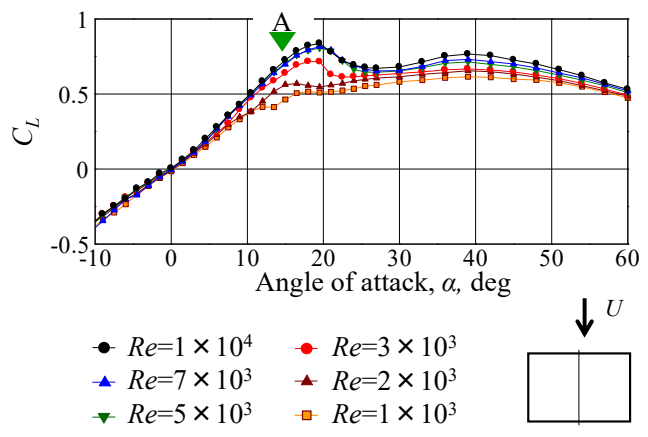

Fig. 8. Lift curves of $A R=1.5$ wing.

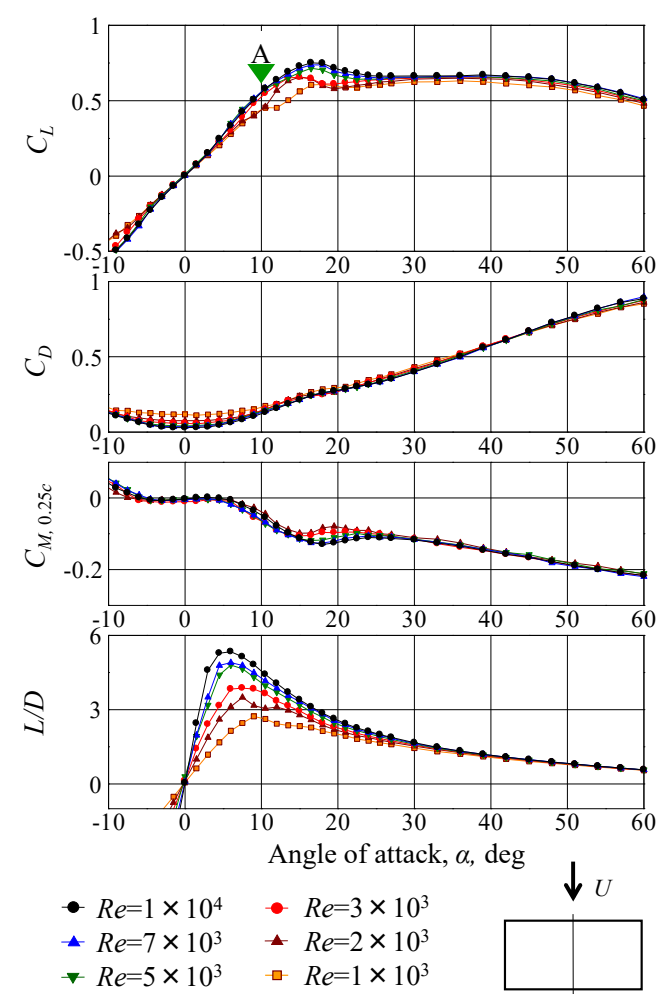

Fig. 9. Aerodynamic characteristics of $A R=2$ wing.

decreases, $C_{L \max }$ decreases at $R e \leq 3 \times 10^{3}$. Although the $C_{L}$ of a large angle of attack decreases as the Reynolds number decreases, the $C_{L}$ of $\alpha=60 \mathrm{deg}$ exhibits a similar value, regardless of the aspect ratio.

As the aspect ratio increases to $A R=2$, the lift slope increases and the lift curve still has $C_{L \max }$ in spite of a thin flat

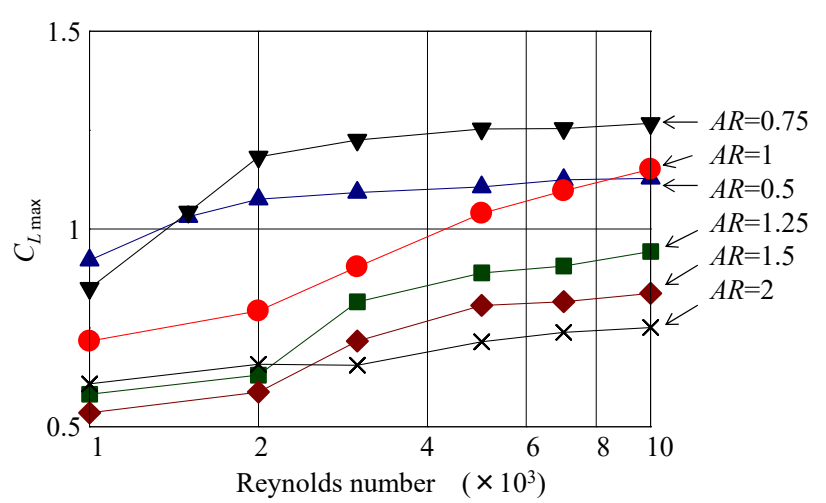

Angle of attack range for determining $C_{L \max }$ $A R \leq 1$ wings : $\alpha \leq 60 \mathrm{deg}, A R>1$ wings : $\alpha \leq 25 \mathrm{deg}$

Fig. 10. Reynolds number effects of $C_{L \max }$ for rectangular wings.

plate. The $C_{L}$ of the flat plate wing with a large aspect ratio increases as the angle of attack increases, and the lift curve changes horizontally near $C_{L}=0.7$ without $C_{L \max }$ increase. ${ }^{13)}$ Thus, the $A R=2$ wing has the characteristics of a wing with a low aspect ratio. As the Reynolds number decreases, $C_{L \max }$ also decreases slightly. That is, the phenomenon of decreasing $C_{L}$ seen with the $A R=1$ wing is no longer seen with the $A R=2$ wing.

$(L / D)_{\max }$ increases as the aspect ratio increases due to the decrease in induced drag. Moreover, $C_{M, 0.25 c}$, having a slight positive slope near $\alpha=0 \mathrm{deg}$, decreases as the angle of attack increases, and reaches -0.2 at $\alpha=60 \mathrm{deg}$, regardless of the aspect ratio.

\subsection{Reynolds number effects of $C_{L \max }$}

Figure 10 shows the relationship between $C_{L \max }$ and Reynolds number for the rectangular wings used during the experiments. The aspect ratio of the wing showing a large $C_{L \max }$ is 1 or less. The $A R=1$ wing has a large decrease in $C_{L \max }$ as the Reynolds number decreases. As the aspect ratio of the wing changes, the rate of $C_{L \max }$ decreasing slows. Therefore, the aspect ratios of the wings having the largest $C_{L \max }$ at $R e=1 \times 10^{4}$ and $R e=1 \times 10^{3}$ are 0.75 and 0.5 , respectively.

\subsection{Comparison with theory}

Although the lift slope of a wing with a low aspect ratio is calculated using the lifting surface theory (VLM), the vortex lift added by the tip vortex, as seen with the lift curve of wings with low aspect ratios, cannot be obtained by the potential theory. Polhamus ${ }^{15,16)}$ simulated vortex lift by turning the direction of the suction force acting on the leading-edge of the wing from horizontal to vertical (i.e., Polhamus' Leading-edge Suction Analogy). As for the theoretical lift slope of rectangular wings, Lamar and Gloss ${ }^{21)}$ demonstrated that the vortex lift can be calculated by introducing a suction analogy for leading-edge and side-edge suction forces. The theoretical $C_{L}$ is expressed using the potential lift $C_{L, p}$, the leading-edge vortex lift $C_{L, V l}$, and the side-edge vortex lift $C_{L, V s}$ as follows: 


$$
\left.\begin{array}{rl}
C_{L} & =C_{L, p}+C_{L, V l}+C_{L, V s} \\
& =K_{P} \sin \alpha \cos ^{2} \alpha+\left(K_{V l}+K_{V s}\right) \sin ^{2} \alpha \cos \alpha
\end{array}\right\}
$$

where, $K_{P}$ is the lift slope given by the lifting surface theory (VLM), and $K_{V s}$ and $K_{V l}$ are estimated from the leadingedge and side-edge suction force, respectively. Lamar and Gloss showed these coefficients in the aspect ratio of the wing, and compared them with the experimental results at high Reynolds numbers.

Furthermore, the $C_{D}$ of the thin flat-plate is determined using $C_{L}$ as follows:

$$
C_{D}=C_{L} \tan \alpha+C_{D 0},
$$

where, $C_{D 0}$ corresponds to the minimum drag coefficient $C_{D \text { min }}$, which is approximately twice the skin friction drag $C_{f}$.

Figures 11(a)-(d) present a comparison between the theoretical and experimental values of $C_{L}$ and $C_{D}$. As shown in Fig. 11(a), the lift curve of the $A R=1$ wing almost corresponds to the theoretical results at $R e=1 \times 10^{4}$, and the lift curve of $R e=1 \times 10^{3}$ approaches the theoretical $C_{L}$ obtained by subtracting the suction force of the side-edge. The experimental $C_{D}$ of the $A R=1$ wing is also close to the theoretical $C_{D}$. The experimental $C_{D}$ corresponds to the tangential component of theoretical $C_{L}$ with and without vortex lift at $R e=1 \times 10^{4}$ and $R e=1 \times 10^{3}$, respectively. As the aspect ratio drops below 1 for the $A R=0.5$ wing (Fig. 11(b)), the lift curve of $R e=1 \times 10^{4}$ corresponds to the theoretical lift curve. However, the $C_{L}$ of $R e=1 \times 10^{3}$ is larger than the theoretical $C_{L}$ obtained by subtracting the side-edge vortex lift. That is, the decreasing $C_{L}$ due to the disappearance of vortex lift is hardly noticeable with the $A R<1$ wing.

As the aspect ratio increases above $1(A R=1.5$ and 2 wings, Figs. 11(c) and (d), respectively), the experimental and theoretical lift slopes match only in the small angle of attack range at both $R e=1 \times 10^{3}$ and $R e=1 \times 10^{4}$. However, the non-linear lift slopes at $R e=1 \times 10^{4}$ cannot be explained using the present method. Furthermore, the experimental $C_{D}$ only corresponds theoretically in the small angle of attack range. The non-linear lift slope of these wings only corresponds theoretically in the small angle of attack range. The non-linear lift slope of these wings probably appears due to the large vortex generated at the leading-edge of the wing at low Reynolds numbers, as shown in the flow visualizations.

From these results, it is clear that although the vortex lift is added on the lift slope of the $A R=1$ wing until the large angle of attack at $R e=1 \times 10^{4}$, its angle of attack range decreases as the Reynolds number decreases.

\subsection{Flow visualization around the wing}

Figures 12(a)-(d) show the tip vortex in the vertical plane of the trailing-edge as seen from the back of the wing. These photos are the tip vortices at the angle of attack where the difference in $C_{L}$ is large at $R e=2 \times 10^{3}$ and $R e=5 \times 10^{3}$ : (a) $\alpha=25 \mathrm{deg}$ for the $A R=1$ wing, (b) $\alpha=30 \mathrm{deg}$ for

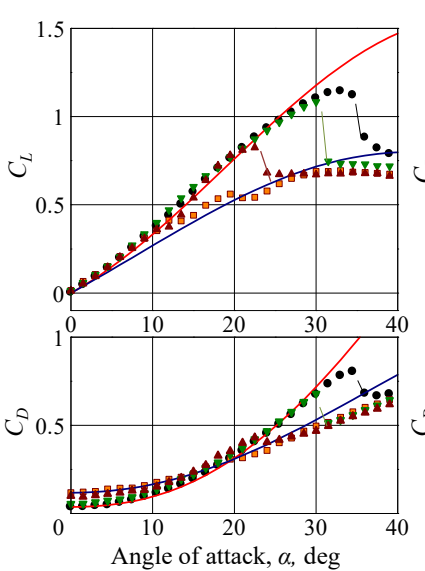

(a) $A R=1$

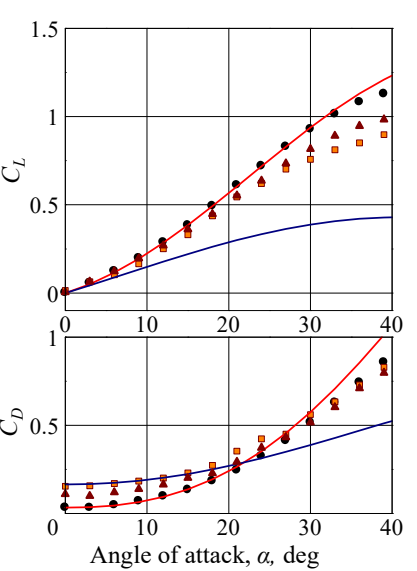

(b) $A R=0.5$

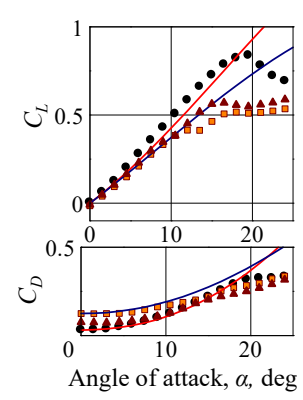

(c) $A R=1.5$

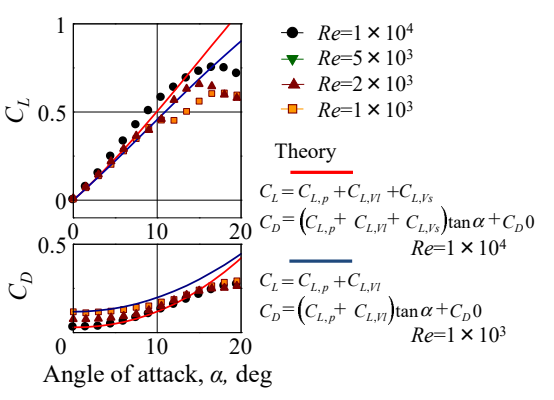

(d) $A R=2$
Fig. 11. Comparison between theory and experiment.

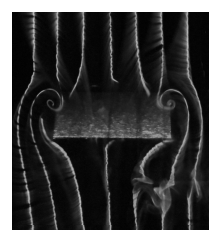

$R e=2 \times 10^{3}$

(a) $A R=1$ wing, $\alpha=25 \mathrm{deg}$
(Point $\mathrm{A}$ in Fig. 4)

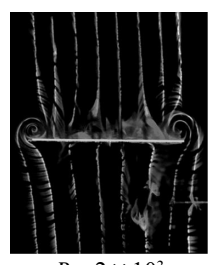

$R e=2 \times 10^{3}$

(Point A in Fig. 8) (c) $A R=1.5$ wing, $\alpha=15 \mathrm{deg}$

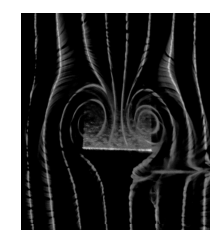

$R e=2 \times 10^{3}$

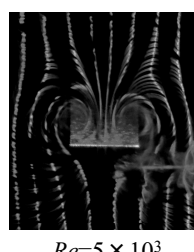

$R e=5 \times 10^{3}$

(b) $A R=0.5$ wing , $\alpha=30 \mathrm{deg}$ (Point A in Fig. 6)

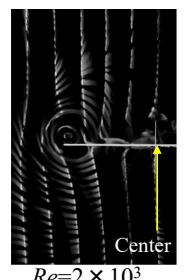

(d) $A R=2$ wing, $\alpha=10 \mathrm{deg}$ (Point A in Fig. 9)

Fig. 12. Tip vortices in the vertical plane of the wing trailing-edge.

the $A R=0.5$ wing, (c) $\alpha=15 \mathrm{deg}$ for the $A R=1.5 \mathrm{wing}$, and (d) $\alpha=10 \mathrm{deg}$ for the $A R=2$ wing. Point $\mathrm{A}$ in the lift curves (seen in Figs. 4, 6, 8, and 9) indicates these angles of attack. As shown in Fig. 12(a), although the position of the tip vortex is just upward of the wing tip at $R e=5 \times 10^{3}$, it separates from the wing surface at $R e=2 \times 10^{3}$. The visualization of tip vortex here corresponds to the Reynolds number effect of $C_{L}$. That is, the disappearance of vortex lift is caused by the separation of tip vortex from the wing surface. 

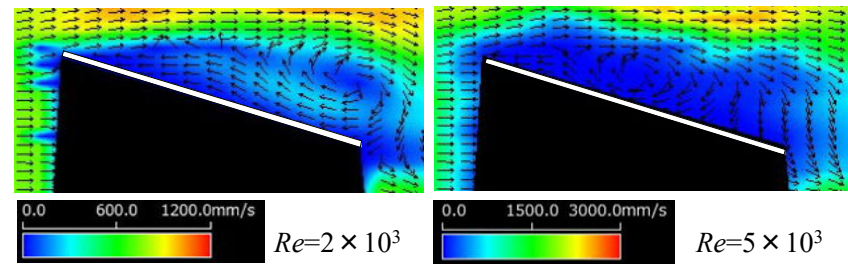

(a) $A R=1.5$ wing, $\alpha=15 \operatorname{deg}$ (Point A in Fig. 8)
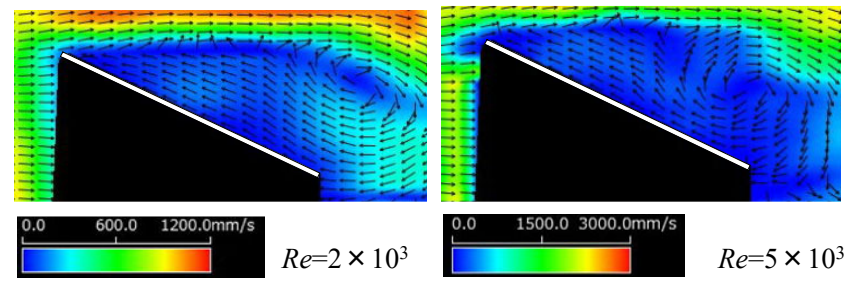

(b) $A R=1$ wing, $\alpha=25 \operatorname{deg}$ (Point A in Fig. 4)

Fig. 13. Velocity vectors on the center chord of the wings with $A R=1.5$ and $A R=1$

Figure 12(b) shows that the position of the tip vortex is just upward of the wing tip for both $R e=2 \times 10^{3}$ and $R e=5 \times 10^{3}$. The distance between the tip vortices of both wing tips is smaller than that of the $A R=1$ wing. In other words, the disappearance of vortex lift of the $A R=0.5$ wing is hardly noticeable, because of the small wing-width.

Figures 12(c) and (d) show that the position of the tip vortex is just upward of the wing tip, and is the same for both $R e=2 \times 10^{3}$ and $R e=5 \times 10^{3}$, even though $C_{L}$ changes. The influence of tip vortex is small in wings with higher aspect ratios. To verify that the difference in the $C_{L}$ of the $A R>1$ wing was due to a lower Reynolds number, flow visualizations of the chord-wise direction at the center of the wingspan were performed. Because the streamline using the smoke-wire method could not clarify the difference in Reynolds number, the PIV method was used. Figure 13(a) shows the flow of the chord-wise direction at the center of the $A R=1.5$ wing with $\alpha=15 \mathrm{deg}$. As shown in the flow at $R e=5 \times 10^{3}$ (right figure), the vortex on the upper surface of the wing is seen at the middle position of the chord, and the downward flow at the back of the vortex is seen on the wing surface. The vortex generated from the leadingedge grows at the middle of the wing and flows to the back of the wing. As shown in the flow at $R e=2 \times 10^{3}$ (left figure), the vortex on the upper surface of the wing is seen at the latter part of the wing, and the downward flow of the vortex is seen at the back of the wing. The vortex generated by the flow separation at the leading-edge of the wing flows to a distant location on the wing surface, and increases at the latter part of the wing. The growing vortex flows to the back of the wing periodically.

Figure 13(b) shows the chord-wise directional flow at the center of the $A R=1$ wing with $\alpha=25 \mathrm{deg}$. The vortices are seen at a position away from the upper surface of the wing for both $\operatorname{Re}=2 \times 10^{3}$ and $\operatorname{Re}=5 \times 10^{3}$. Although the vortex at $R e=5 \times 10^{3}$ is quite close to the wing, the upper surface flow is reversed. The flow of the center chord wing is the separation flow in spite of the Reynolds number. That is, the be- havior of the tip vortex largely affects the Reynolds number effects of the $A R=1$ wing.

From these flow visualizations, it is recognized that the Reynolds number effects of $A R \leq 1$ and $A R>1$ wings are influenced by the behavior of the tip vortices and that of chord-wise directional vortex on the surface of the wing section, respectively.

\section{Conclusion}

To obtain the aerodynamic characteristics of rectangular wings having aspect ratios varying from 0.5 to 2 at low Reynolds numbers corresponding to an insect-sized wing, lowpressure wind tunnel tests were conducted at Reynolds number ranging between $1 \times 10^{3}$ and $1 \times 10^{4}$. The main findings are listed below.

1) The Reynolds number effects on the aerodynamic characteristics of wings with low aspect ratios using the present Reynolds numbers are large in a specific aspect ratio wing.

2) Although $C_{L \max }$ of the rectangular wing with $A R=1$ shows higher values at large angles of attack at $R e=1 \times 10^{4}$, it decreases quickly as the Reynolds number decreases to $R e=1 \times 10^{3}$ due to the disappearance of vortex lift.

3) The disappearance of vortex lift is caused by separation of the tip vortex from the wing surface.

4) As the aspect ratio decreases below $1, C_{L \max }$ of the wing is as large as that of the $A R=1$ wing, and that of the $A R=0.5$ wing hardly decreases as the Reynolds number decreases.

5) As the aspect ratio increases above $1, C_{L \max }$ and the Reynolds number effects on the wing decrease.

6) The main cause of the Reynolds number effects is the tip vortices for wings with $A R \leq 1$ and a vortex of chordwise direction for wings with $A R>1$.

\section{Acknowledgments}

The authors are grateful to Dr. Akira Azuma (Professor Emeritus of the University of Tokyo) for his useful advice and warm encouragement. We also thank Mr. Hiroki Maruoka (postgraduate of Kanazawa Institute of Technology) for his assistance in this study.

This work was supported by KAKENHI (25630395 and 16K06894).

\section{References}

1) Pines, D. J. and Bohorquez, F.: Challenges Facing Further Micro-AirVehicle Development, J. Aircraft, 43, 2 (2006), pp. 290-305.

2) Wood, R. J., Finio, B., Karpelson, M., Ma, K., Perez-Arancibia, N. O., Sreetharan, P. S., Tanaka, H., and Whitney, J. P.: Progress on Pico Air Vehicles, Int. J. Robot. Res., 31, 11 (2012), pp. 1292-1302.

3) Oyama, A. and Fuji, K.: A Study on Airfoil Design for Future Mars Airplane, AIAA-2006-1484, 44th AIAA Aerospace Sciences Meeting and Exhibit, 2006.

4) Okamoto, M., Yasuda, K., and Azuma, A.: Aerodynamic Characteristics of the Wings and Body of a Dragonfly, J. Exp. Biol., 199 (1996), pp. 281-294.

5) Azuma, A., Okamoto, M., and Yasuda, K.: Aerodynamic Characteristics of Wing at Low Reynolds Number, Fixed and Flapping Wing 
Aerodynamics for Micro Air Vehicle Applications, Mueller, T. J. (ed.), Progress in Astronautics and Aeronautics, Vol. 195, AIAA, Reston, VA, 2001, pp. 341-398.

6) Kesel, A. B.: Aerodynamic Characteristics of Dragonfly Wing Sections Compared with Technical Aerofoils, J. Exp. Biol, 203 (2000), pp. 2125-2135.

7) Sunada, S., Yasuda, T., Yasuda, K., and Kawachi, K.: Comparison of Wing Characteristics at an Ultralow Reynolds Number, J. Aircraft, 39, 2 (2002), pp. 331-338.

8) Obata, A. and Shinohara, S.: Flow Visualization Study of the Aerodynamics of Modeled Dragonfly Wings, AIAA J., 47, 12 (2009), pp. 3043-3047.

9) Shi, S. X., Liu, Y. Z., and Chen, J. M.: An Experimental Study of Flow around a Bio-inspired Airfoil at Reynolds Number $2.0 \times 10^{3}, \mathrm{~J}$. Hydrodynamics, 24, 3 (2012), pp. 410-419.

10) Okamoto, M. and Ebina, K.: Effectiveness of Large-Camber Circular Arc Airfoil at Very Low Reynolds Numbers, Trans. Jpn. Soc. Aeronaut. Space Sci., 59 (2016), pp. 295-304.

11) Ohtake, T., Nakae, Y., and Motohashi, T.: Nonlinearity of the Aerodynamic Characteristics of NACA0012 Aerofoil at Low Reynolds Numbers, J. Jpn. Soc. Aeronaut. Space Sci., 55 (2007), pp. 439-445. (in Japanese).

12) Torres, G. E. and Mueller, T. J.: Aerodynamic Characteristics of Low Aspect Ratio Wings at Low Reynolds Numbers, Fixed and Flapping Wing Aerodynamics for Micro Air Vehicle Applications, Mueller, T. J. (sd.), Progress in Astronautics and Aeronautics, Vol. 195, AIAA, Reston, VA, 2001, pp. 115-141.

13) Okamoto, M. and Azuma, A.: Aerodynamic Characteristics at Low Reynolds Numbers for Wings of Various Planforms, AIAA J., 49, 6 (2011), pp. 1135-1150.

14) Mizoguchi, M. and Itoh, H.: Effect of Aspect Ratio on Aerodynamic
Characteristics at Low Reynolds Numbers, AIAA J., 51, 7 (2013), pp. 1631-1639.

15) Polhamus, E. C.: A Concept of the Vortex Lift of Sharp-Edge Delta Wings Based on a Leading-Edge-Suction Analogy, NASA TN-D3767, 1966.

16) Polhamus, E. C.: Application of the Leading-Edge-Suction Analogy of Vortex Lift to the Drag Due to Lift of Sharp-Edge Delta Wings, NASA TN D-4739, 1968.

17) Okamoto, M., Sasaki, D., Sasaki, K., and Nakamura, T.: Aerodynamic Characteristics of Insect Sized AR=1 Rectangular Wing, J. Jpn. Soc. Aeronaut. Space Sci., 65 (2017), pp. 177-183 (in Japanese).

18) Sasaki, D., Miwa, T., Yamaguchi, Y., Natsume, Y., Iwafune, T., Fujii, R., Akasaka, T., Okamoto, M., Takahashi, S., Misaka, T., Obayashi, S., and Shimoyama, K.: Experimental and Numerical Investigation of Flow Phenomena Associated with Low-Reynolds Number Flow, Proceedings of the Eighteenth International Symposium on Advanced Fluid Information, pp. 22-23, Sendai, Japan, November 2018.

19) Okamoto, M., Sasaki, D., Sasaki, K., and Nakamura, T.: Planform Aerodynamic Characteristics of Insect Sized Wings, JAXA-SP-18005, 2019, pp. 195-198 (in Japanese).

20) Sindo, S.: Low-speed Wind Tunnel Testing Technique, Corona Publishing, Co., Ltd., Tokyo, 1992 (in Japanese).

21) Lamar, J. E. and Gloss, B. B.: Subsonic Aerodynamic Characteristics of Interaction Lifting Surfaces with Separated Flow around Sharp Edges Predicted by a Vortex-Lattice Method, NASA TN D-7921, 1975 .

Naofumi Ohnishi Associate Editor 